\title{
METRONOMIC CHEMOTHERAPY: ROLE IN DEVELOPING COUNTRIES FOR HEAD AND NECK CANCER
}

Head and neck squamous cell carcinoma (HNSCC) is one of the countries. ${ }^{[1]}$ More than half of the patients present in advanced stage. ${ }^{[2,3]}$ The number of institutes providing cancer care is highly inadequate in developing countries. A cross-sectional study on head and neck cancers (HNC) showed that various beliefs ('cancer is a curse'), nonavailability of transport, believing mouth ulcer to be selflimiting and prolonged treatment resulting in family stress are important factors in treatment delay. ${ }^{[4]}$ Other reasons included poor socioeconomic status of the patient, cost of care and high rate of illiteracy. ${ }^{[5]}$ The current pricing system of chemotherapy drugs is unsustainable and not affordable for many patients on palliative care. There is an enormous financial pressure on the society, especially in developing countries due to the skyrocketing costs. ${ }^{[6]}$ In these patients, already in advanced stage of cancer, poor access to the specialized cancer centres is further compounded with increasingly long waiting lists for treatment. A prospective observational study on $201 \mathrm{HNC}$ patients showed that there was a delay of 6 months from the onset of symptoms to final treatment. ${ }^{[7]}$ Thus, there was a need to initiate a therapy that might inhibit progression of tumour and keep patients operable during this delay. The therapy should have minimal toxicity, economical and easy to administer which becomes very important for low- to middle-income developing Asian countries.

Metronomic chemotherapy (MC) is one such emerging therapeutic option in clinical oncology. The term 'metronomic' is derived from the musical device 'metronome' that produces an audible beat - a click or other sound - at regular intervals. In the same way, MC is the regular administration of low-dose chemotherapy $\left(1 / 10^{\text {th }}-1 / 3^{\text {rd }}\right.$ of the maximum tolerated dose $)$ that results in a constant low blood level of the drug. ${ }^{[8]}$

MC exerts its anticancer activity by inhibiting tumour angiogenesis, stimulating anticancer immune response

Correspondence: Dr. Deepa Nair,

Department of Head and Neck Oncology,

Tata Memorial Centre, Mumbai, Maharashtra, India.

Email: drdeepanair@hotmail.com and inducing tumour dormancy. ${ }^{[8]}$ The $\mathrm{MC}$ antiangiogenic effect was demonstrated by Browder (cyclophosphamidebased MC) and Klement (vinblastine-based MC). ${ }^{[9,10]}$ $\mathrm{MC}$ also acts on the innate and adaptive immunity. It has been shown that MC selectively induced reduction of circulating regulatory T-cells and subsequent reduction of their inhibitory functions on antigen-specific immune response. ${ }^{[11]}$ Tumour dormancy is mainly due to angiogenic dormancy (inability of tumour cells to recruit blood vessels), cellular dormancy (tumour cells in G0G1 arrest) and immune surveillance (prevent residual tumour cells expansion). ${ }^{[8]} \mathrm{MC}$ has been used mainly in the palliative settings; however, it can also been tried in the adjuvant and neoadjuvant settings.

\section{MC in Palliative Settings}

Prospective studies have shown that MC as compared to single-agent cisplatin chemotherapy, in patients with metastatic, relapsed or inoperable $\mathrm{HNC}^{[12,13]}$ may be beneficial. Results showed a significant better progressionfree survival (PFS) (249 days vs. 152 days, $P=0.02$ ) and overall survival (OS) (101 days vs. 66 days, $P=0.014$ ) in $\mathrm{MC}$ than cisplatin-based chemotherapy with better pain control. A retrospective study evaluated the role of MC maxillary sinus cancer patients on palliative treatment. It showed that the estimated median survival in patients with an event-free period after the last therapy of $<6$ months was 45 days, whereas it was 409 days in patients with an event-free period post last therapy $>6$ months $(P=0.063) \cdot{ }^{[14]}$

However, no head-to-head studies have been done comparing $\mathrm{MC}$ to the standard of care drug regimen of TPF/cetuximab in this setting. A retrospective study of 60 patients with metastatic/recurrent head and neck squamous cell cancer treated with weekly paclitaxel $(80 \mathrm{mg} / \mathrm{m} 2)$ and cetuximab were matched with 60 patients treated with oral MC. The median OS was 191 days in metronomic cohort and 256 days in cetuximab cohort. The hazard ratio was 0.58 in favour of cetuximab cohort $(P=0.031){ }^{[15]}$ However, cetuximab, being a significantly more expensive therapy, may not be feasible routinely 
in low- and middle-resource countries such as India and Pakistan. Complete remission was seen in two patients, partial remission in seven patients, stable disease in four patients, and progressive disease in two patients in a single-arm prospective observational study of 15 patients who received $\mathrm{MC}$ in the form of oral methotrexate (15 mg/m²/week), oral celecoxib (200 mg twice daily) and erlotinib (150 mg once daily). The median estimated PFS was 148 days. Six patients (40\%) showed Grade III/ IV toxicity. Thus, addition of erlotinib showed promising results in improving PFS, but proper selection of patients is essential as $40 \%$ had Grade III/IV toxicity. ${ }^{[16]}$ Mateen et al. ${ }^{[17]}$ presented their experience with $\mathrm{MC}$ in recurrent HNSCC. A total of 72 patients were enrolled and were prescribed oral methotrexate $2.5 \mathrm{mg}$ twice in a week and capecitabine $500 \mathrm{mg}$ twice a day, for at least 6 months. 2-year PFS and OS were 18\% and 40\%, respectively. Partial response was seen in 17 (29\%) patients while 31 (54\%) patients showed stable disease at 6-month followup. However, this is preliminary data and the complete article is yet too published.

\section{MC in the Adjuvant Setting for HNC}

The use of MC in the adjuvant setting is still investigational. Pandey et al. ${ }^{[18]}$ published a retrospective study of 225 patients with locally advanced operable oral cavity carcinoma (Stage III/IVA) where adjuvant MC \{oral methotrexate $\left(15 \mathrm{mg} / \mathrm{m}^{2}\right)$ once a week and celecoxib (200 mg twice a day) daily\} was given in 130/225 (58\%) patients after completion of the standard treatment for a duration of 18 months. The use of MC improved OS (14 months vs. 26 months, $P=0.04$ ) and disease-free survival (DFS) (8 months vs. 4 months, $P=0.22$ ). None of the patients discontinued MC due to toxicity. Lam et al. reported that the first prospective randomized trial in which a trend of better control of the distant metastasis was observed in the adjuvant MC (29 patients: Levamisole/uracil-tegafur [UFT] therapy) group (10\% vs. $32 \%$ for the control group [34 patients]). However, 5-year disease-free actuarial survival rates for patients with and without adjuvant MC were $57 \%$ and $39 \%$, respectively $(P=0.207) .{ }^{[19]}$ Fujii et al..$^{[20]}$ also reported that adjuvant chemotherapy with UFT was efficacious for treating maxillary sinus carcinoma. The 5-year survival was $76.2 \%$ for the UFT group and $17.9 \%$ for the control group. An on-going non-randomized Phase II study in
Taiwan (NCT00855881) will look into the effect of 1-year maintenance treatment of metronomic tegafur-uracil in HNSCC.

\section{MC in Neoadjuvant Setting}

Studies have shown that MC strategy was associated with a reasonable response rate in neoadjuvant settings for breast cancers. ${ }^{[21]}$ However, the role of MC in neoadjuvant setting for $\mathrm{HNC}$ is yet to be studied. A randomized controlled trial assessing addition of neoadjuvant and maintenance oral MC to standard surgery and adjuvant therapy in Stage III/IV operable oral cancers is ongoing (CTRI no. CTRI/2015/01/005405). The primary endpoint of this study is disease-free survival at 3 years. The secondary endpoint is to assess the safety, toxicity and tumour response to neoadjuvant MC.

Thus, clinical studies have shown that $\mathrm{MC}$ is a promising strategy in the management of locally advanced headneck cancers. It may be especially relevant to patients in the developing countries as it is economical, easy to administer without the need for dedicated oncology setups or day-care centres. Patients with advanced head-neck cancers, especially oral cancers tend to be nutritionally deprived, cachectic and may not tolerate conventional chemotherapy easily. Treatment with MC is an attractive option in these patients who would otherwise have been deemed only for symptomatic, palliative care. Overall, in unresectable or recurrent or metastatic $\mathrm{HNC}, \mathrm{MC}$ has shown good disease control rates with good quality of life outcomes. It should be considered, especially when standard chemotherapy (TPF/cetuximab) may not be feasible due to fitness, logistic or economic issues.

The role of $\mathrm{MC}$ in adjuvant setting has shown promising results in clinical trials and can be considered for maintenance therapy. Combination of MC with other therapies might help in improving response rates. The role of maintenance $\mathrm{MC}$ in patients who undergo salvage surgery also needs validation. Several clinical trials under design or in progress will clarify the best dose, schedule, optimal sequence and timing of administration. There is a number of $\mathrm{MC}$ regimen available which need further standardization. Nevertheless, there is no unanimous consensus for the use of one MC regimen over the other, due to the small study population studies. The role of 
$\mathrm{MC}$ in sensitizing the tissue to radiation is also yet to be studied.

\section{Deepa Nair, Manish Mair, Pankaj Chaturvedi Department of Head and Neck Oncology, Tata Memorial Centre, Mumbai, Maharashtra, India Received: 3 March 2017/Accepted: 14 March 2017}

\section{References}

1. Sankaranarayanan R, Masuyer E, Swaminathan R, et al. Head and neck cancer: A global perspective on epidemiology and prognosis. Anticancer Res 1998;18:4779-86.

2. Agarwal AK, Sethi A, Sareen D, et al. Treatment delay in oral and oropharyngeal cancer in our population: The role of socio-economic factors and health-seeking behaviour. Indian J Otolaryngol Head Neck Surg 2011;63:145-50.

3. Patel UA, Lynn-Macrae A, Rosen F, et al. Advanced stage of head and neck cancer at a tertiary-care county hospital. Laryngoscope 2006;116:1473-7.

4. Kumar S, Heller RF, Pandey U, et al. Delay in presentation of oral cancer: A multifactor analytical study. Natl Med J India 2001;14:13-7.

5. Chintamani, Tuteja A, Khandelwal R, et al. Patient and provider delays in breast cancer patients attending a tertiary care centre: A prospective study. JRSM Short Rep 2011;2:76.

6. Riaz MK, Bal S, Wise-Draper T. The impending financial healthcare burden and ethical dilemma of systemic therapy in metastatic cancer. J Surg Oncol 2016;114:323-8.

7. Joshi P, Nair S, Chaturvedi P, et al. OP140: Delay in seeking specialist medical care and advanced oral cavity cancer: Experience in a tertiary care centre, India. Oral Oncol 2013;49 Supplement 1:S58.

8. De Felice F, Musio D, Tombolini V. Head and neck cancer: Metronomic chemotherapy. BMC Cancer 2015;15:677.

9. Browder $\mathrm{T}$, Butterfield $\mathrm{CE}$, Kräling $\mathrm{BM}$, et al. Antiangiogenic scheduling of chemotherapy improves efficacy against experimental drug-resistant cancer. Cancer Res 2000;60:1878-86.

10. Klement G, Baruchel S, Rak J, et al. Continuous low-dose therapy with vinblastine and VEGF receptor-2 antibody induces sustained tumor regression without overt toxicity. J Clin Invest 2000;105:R15-24.

11. Ghiringhelli F, Menard C, Puig PE, et al. Metronomic cyclophosphamide regimen selectively depletes $\mathrm{CD} 4+\mathrm{CD} 25+$ regulatory $\mathrm{T}$ cells and restores $\mathrm{T}$ and $\mathrm{NK}$ effector functions in end stage cancer patients. Cancer Immunol Immunother 2007;56:641-8.

12. Patil VM, Noronha V, Joshi A, et al. A prospective randomized phase II study comparing metronomic chemotherapy with chemotherapy (single agent cisplatin), in patients with metastatic, relapsed or inoperable squamous cell carcinoma of head and neck. Oral Oncol 2015; 51:279-86.

13. Noronha V, Joshi A, Marfatia S, et al. Health-related quality of life in patients with metastatic, relapsed, or inoperable squamous cell carcinoma of the head and neck in India. Support Care Cancer 2016;24:1595-602.

14. Patil VM, Noronh V, Joshi A, et al. Metronomic palliative chemotherapy in maxillary sinus tumor. South Asian J Cancer 2016;5:56-8.

15. Parikh PM, Hingmire SS, Deshmukh CD. Selected current data on metronomic therapy (and its promise) from India. South Asian J Cancer 2016;5:37-47.

16. Patil VM, Chakraborty S, Jithin TK, et al. An audit of the results of a triplet metronomic chemotherapy regimen incorporating a tyrosine kinase inhibitor in recurrent/ metastatic head and neck cancers patients. South Asian J Cancer 2016;5:48-51.

17. Mateen A, Adil AR, Maken RN, et al. Metronomic chemotherapy in recurrent head and neck cancer. Clin Oncol 2015;33:e17007. Available from: http://www. meetinglibrary.asco.org/content/144149-156. [Last cited on 2017 Feb 26].

18. Pandey A, Desai A, Ostwal V, et al. Outcome of operable oral cavity cancer and impact of maintenance metronomic chemotherapy: A retrospective study from rural India. South Asian J Cancer 2016;5:52-5.

19. Lam P, Yuen AP, Ho CM, et al. Prospective randomized study of post-operative chemotherapy with levamisole and UFT for head and neck carcinoma. Eur J Surg Oncol 2001;27:750-3.

20. Fujii M, Ohno Y, Tokumaru Y, et al. Adjuvant chemotherapy with oral tegaful and uracil for maxillary sinus carcinoma. Oncology 1998;55:109-15.

21. Petry V, Gagliato DM, Leal AI, et al. Metronomic chemotherapy in the neoadjuvant setting: Results of two parallel feasibility trials (TraQme and TAME) in patients with HER2+ and HER2-locally advanced breast cancer. Braz J Med Biol Res 2015;48:479-85. 